\title{
Changes in the Ångstrom Exponent during Aerosol Coagulation and Condensation
}

\author{
Chang H. Jung*, Ji Yi Lee ${ }^{1)}$ and Yong P. Kim²) \\ Department of Health Management, Kyungin Women's College, 101 Gyesan-gil, Gyeyang-gu, Incheon 407-740, Korea \\ ${ }^{1)}$ Department of Environmental Engineering, Chosun University, 309 Pilmun-daero, Dong-gu Gwangju 501-759, Korea \\ ${ }^{2)}$ Department of Environmental Science and Engineering, Ewha Womans University, 11-1 Daehyun-dong, Seodaemun-gu, \\ Seoul 120-750, Korea
}

*Corresponding author. Tel: +82-32-540-0166, E-mail: jch@kic.ac.kr

\begin{abstract}
In this study, the Angstrom exponent for polydispersed aerosol during dynamic processes was investigated. Log-normal aerosol size distribution was assumed, and a sensitivity analysis of the Angstrom exponent with regards the coagulation and condensation process was performed. The Angstrom exponent is expected to decrease because of the particle growth due to coagulation and condensation. However, it is difficult to quantify the degree of change. In order to understand quantitatively the change in the Angstrom exponent during coagulation and condensation, different real and imaginary parts of the refractive index were considered. The results show that the Angstrom exponent is sensitive to changes in size distribution and refractive index. The total number concentration decreases and the geometric mean diameter of aerosols increase during coagulation. On the while, the geometric standard deviation approaches monodispersed size distribution during the condensation process, and this change in size distribution affects the Angstrom exponent. The degree of change in the Angstrom exponent depends on the refractive index and initial size distribution, and the size parameter changes with the Angstrom exponent for a given refractive index or chemical composition; this indicates that the size distribution plays an important role in determining the Angstrom exponent as well as the chemical composition. Subsequently, this study shows how the Angstrom exponent changes quantitatively during the aerosol dynamics processes for a log-normal aerosol size distribution for different refractive indices; the results showed good agreement with the results for simple analytic size distribution solutions.
\end{abstract}

Key words: Aerosol size distribution, Coagulation, condensation, Ångstrom exponent, Polydispersed aerosol, Aerosol optical properties

\section{INTRODUCTION}

The Ångstrom exponent expresses the spectral dependence of aerosol optical thickness $(\tau)$ on the wavelength of incident light $(\lambda)$. The Ångstrom exponent provides information on the particle size (the larger the exponent, the smaller is the particle size), aerosol phase function, and the relative magnitude of aerosol radiances at different wavelengths. It can be used for computing aerosol optical depth at different wavelengths and characterizing the aerosol types as well as in radiative transfer modeling and Earth Radiation Budget Study. The Angstrom exponent is often used as a qualitative indicator of aerosol particle size. It contains information on intensive aerosol properties such as size distribution and refractive index. Different size distributions such as power law, gamma, and log-normal distributions have been used to model atmospheric aerosols (Schuster et al., 2006).

Because of the great variability in the physical, chemical, and optical properties of aerosols, it is difficult to develop a simple and generalized atmospheric aerosol model. In particular, it is important to simulate the aerosol optical properties for estimating radiative forcing taking into account the aerosol optical depth, single scattering albedo, asymmetry parameter, and the Angstrom exponent. In simulating the Ångstrom exponent, it is necessary to have size and chemical composition data (O'Neill et al., 2001a, b).

Atmospheric formation and removal of particles are governed by a number of complex dynamic processes including nucleation, condensation, coagulation, chemical transformation among the gas/particle phases, and deposition.

The size and composition of atmospheric aerosols change continuously during the dynamic processes such as coagulation or condensation. For example, aerosol particles collide because of their random mo- 
tions and coalesce to form larger chains of flocculation made up of many particles. The Brownian motion of particles, turbulence, presence of a shear field, and external forces such as gravity and electrical forces could cause coagulation. Condensation is the process by which an aerosol particle grows with the accretion of monomers or individual molecules to an already existent particle. This means that the change in the size distribution due to coagulation and condensation process affect the Angstrom exponent and the exponent may change as a function of time owing to the aerosol dynamic processes. Hence, aerosol dynamics plays an important role in the estimation of optical properties such as the Ångstrom exponent. Although the Ångstrom exponent is expected to decrease during coagulation and condensation because of aerosol growth, it is difficult to quantify the degree of change.

In this study, the Ångstrom exponent for polydispersed aerosol during dynamic processes was simulated. Log-normal aerosol size distribution was assumed, and the moment method was used. Analytic solutions for coagulation and condensation were used, and their results were compared with those of the numerical solution for estimating the Ångstrom exponent. Finally, this study shows the results of the sensitivity analysis for the Angstrom exponent as a function of size parameter and refractive index under unimodal aerosol size distribution.

\section{THE ÅNGSTROM EXPONENT DURING COAGULATION AND CONDENSATION PROCESSES}

In many cases, the size distribution of ambient polydispersed aerosol can be represented by the log-normal distribution function (Seinfeld and Pandis, 1998):

$$
n\left(\ln d_{p}\right)=\frac{N_{p}}{3 \sqrt{2 \pi} \ln \sigma_{g}} \exp \left[-\frac{-\ln ^{2}\left(d_{p} / d_{g}\right)}{2 \ln ^{2} \sigma_{g}}\right]
$$

where $d_{p}$ is the diameter of the particle; $d_{g}$, the geometric mean diameter; $\sigma_{g}$, the geometric standard deviation; and $N$, the total number concentration.

The $k$ th moment can be written using the moment equations for a log-normal distribution as follows (Binkowski and Shankar, 1995; Lee et al., 1984):

$$
\begin{aligned}
& M_{k}=\int_{0}^{\infty} d_{p}^{k} n\left(d_{p}\right) d d_{p}=\int_{-\infty}^{\infty} d_{p}^{k} n\left(\ln d_{p}\right) d\left(\ln d_{p}\right), \\
& d_{g}=\frac{M_{3}^{2 / 3}}{\left(M_{0}^{3} M_{6}\right)^{1 / 6}}, \sigma_{g}=\exp \left\{\sqrt{\frac{1}{9} \ln \left(\frac{M_{0} M_{6}}{M_{3}^{2}}\right)}\right\}
\end{aligned}
$$

where $k$ is an arbitrary integer. Among the $M_{k} s, M_{0}$ re- presents the total number of particles, and $\pi / 6 M_{3}$ the total volume of particles.

Under the assumption that aerosol size distribution remains log-normal, the following relation holds.

$$
M_{k}=M_{0} d_{g}^{k} \exp \left(\frac{k^{2}}{2} \ln ^{2} \sigma\right)
$$

Total aerosol optical thickness can be expressed as follows (Cachorro and Frutos, 1994).

$$
\tau(\lambda)=b_{e x t} \cdot z
$$

where $b_{\text {ext }}$ is the aerosol extinction coefficient, and $z$ is the height of atmospheric layer.

Under the assumption of the homogeneous atmospheric layer, the Ångstrom exponent $(\alpha)$ can be expressed as follows (Jung and Kim, 2010; Seinfeld and Pandis, 1998).

$$
\alpha=-\frac{\log \left(b_{e x t 1} / b_{e x t 2}\right)}{\log \left(\lambda_{1} / \lambda_{2}\right)}
$$

Here, $b_{\text {ext }}$ is the overall extinction coefficient, and $\lambda$ is the wavelength, which can be expressed as follows (Jung and Kim, 2008; Seinfeld and Pandis, 1998). The Angstrom exponent is the slope of the graph showing the wavelength dependence of the aerosol optical depth (extinction coefficient) in logarithmic coordinates and can be efficiently used in describing aerosol size.

$$
b_{e x t}=\int_{0}^{d_{p}^{\max }} \frac{\pi d_{p}^{2}}{4} Q_{e x t}\left(d_{p}, \lambda, m\right) n\left(d_{p}\right) d d_{p}
$$

Here, $Q_{\text {ext }}\left(d_{p}, \lambda, m\right)$ is the single particle extinction efficiency for particles of diameter $\left(d_{p}\right)$ and for light with wavelength $(\lambda)$ and refractive index $(m) ; n\left(d_{p}\right)$ is the size distribution. In this study, two wavelengths450 and $670 \mathrm{~nm}$-were used for calculating the Angstrom exponent.

As shown in Eq. (5), the Ångstrom exponent can be calculated from the extinction coefficient. It can be expressed in the formula that is usually used to describe the dependency of the aerosol optical thickness or aerosol extinction coefficient on wavelength.

Although the Ångstrom exponent can be calculated from the extinction coefficient, the change in the extinction coefficient during coagulation and condensation is insufficient to understand the change in the Ångstrom exponent.

\section{1 An Analytic Solution for Coagulation and Condensation in the Continuum Regime}

The general governing equation for aerosol coagulation and condensation can be expressed using the moment formula as follows (Jung and Kim, 2006). 


$$
\frac{d M_{k}}{d t}=\operatorname{Coag}_{k}+\operatorname{Con}_{k}
$$

Here, Coag $_{k}$ and Con $_{k}$ represent the coagulation and condensation processes, respectively, and $k$ stands for the $k$ th moment.

By substituting for $k=0,3,6$ in Eq. (7) and using these three ordinary differential equations of moment relation, the change in the total number of particles $(N)$, the numerical solutions for geometric standard deviation $\left(\sigma_{g}\right)$, and the geometric mean diameter $\left(d_{g}\right)$ can be obtained as a function of time (Jung and Kim, 2006; Jung et al., 2004, 2002). The Runge-Kutta $4^{\text {th }}$ order method can be applied.

Many efforts have been made to derive an analytic expression for the time evolution of aerosol size distribution during coagulation and condensation (Jung and Kim, 2006; Jung et al., 2001; Lee et al., 1984; Lee, 1983). Among them, Lee (1983) obtained the change in particle size distribution due to Brownian coagulation in the continuum regime analytically under the assumption of log-normal aerosol size distribution.

The obtained analytic solution can be expressed as follows:

$$
\begin{aligned}
& \ln ^{2} \sigma_{g}=\frac{1}{9} \ln \left[2+\frac{\exp \left(9 \ln ^{2} \sigma_{g 0}\right)-2}{1+\left[1+\exp \left(\ln ^{2} \sigma_{g 0}\right) K N_{0} t\right]}\right] \\
& \frac{d_{g}}{d_{g 0}}=\left\{\frac{\exp \left(\frac{2}{9} \ln ^{2} \sigma_{g 0}\right)\left\{1+\left[1+\exp \left(\ln ^{2} \sigma_{g 0}\right)\right] K N_{0} t\right\}}{\left[2+\frac{\left\{\exp \left(9 \ln ^{2} \sigma_{g 0}\right)-2\right\}}{\left\{1+\left[1+\exp \left(\ln ^{2} \sigma_{g 0}\right)\right] K N_{0} t\right\}}\right]^{1 / 2}}\right\}^{1 / 3}
\end{aligned}
$$$$
\frac{N}{N_{0}}=\frac{1}{1+\left[1+\exp \left(\ln ^{2} \sigma_{g 0}\right)\right] K N_{0} t}
$$

where $K$ is the collision coefficient $(=2 k T / 3 \mu) ; k$, the Boltzmann constant; $T$, the absolute temperature; and $\mu$, the gas viscosity; the subscript 0 stands for the initial conditions $(\mathrm{t}=0)$.

The geometric mean diameter, geometric standard deviation, and total number of particles can be expressed as a function of time according to Lee (1983) under the assumption of the geometric mean diameter does not deviate greatly from the initial geometric mean diameter.

More detailed procedure in obtaining analytic solution for coagulation (Eq. (8)) can be referred in the previous studies (Lee et al., 1984; Lee, 1983).

Likewise, for the condensation process, the moment relations can be usually expressed in terms of the $0 t h$, $l^{\text {st }}$, and $2 n d$ moments (Park et al., 2001) as follows:

$$
\begin{aligned}
& \frac{d M_{0}}{d t}=0, \quad \frac{d M_{3}}{d t}=C(S-1) M_{1}, \frac{d M_{6}}{d t}=\frac{C}{3}(S-1) M_{4} \\
& C=\left[\frac{\rho R T}{M D P_{s}\left\{1+(S+1) P_{s} /(2 p)\right\}}+\frac{\rho L^{2} M}{R K T^{2}}\right]
\end{aligned}
$$

where, $S$ is the saturation ratio; $R$, the gas constant; $T$, the absolute temperature; $M$, the molecular mass of the condensing vapor; $D$, the diffusion coefficient; $p_{s}$, the saturation vapor pressure; $p$, the total gas pressure; $L$, the latent heat; $K$, the thermal conductivity; and $\rho$, the particle density.

From Eq. (9), the following analytic solution can be obtained by applying the above moment relation to the condensational equation (Park et al., 2001).

$$
\begin{aligned}
& \ln ^{2} \sigma= \\
& \frac{1}{6} \ln \left[1+\frac{\exp \left(6 \ln ^{2} \sigma_{0}\right)-1}{\left\{1+(2 / 3)(6 / \pi) \exp \left(-3 \ln ^{2} \sigma_{0}\right) C(S-1) d_{g 0}^{-2} t\right\}^{2}}\right] \\
& \frac{d_{g}}{d_{g 0}}= \\
& \left\{\frac{\exp \left(9 \ln ^{2} \sigma_{0} / 2\right)\left\{1+(2 / 3)(6 / \pi)^{2 / 3} \exp \left(-3 \ln ^{2} \sigma_{0}\right) C(S-1) d_{g 0}^{-2} t\right\}^{3 / 2}}{\left[\frac{1+\left\{\exp \left(6 \ln ^{2} \sigma_{0}\right)-1\right\}}{\left\{1+(2 / 3)(6 / \pi)^{2 / 3} \exp \left(-3 \ln ^{2} \sigma_{0}\right) C(S-1) d_{g 0}^{-2} t\right\}^{2}}\right]^{3 / 4}}\right\}^{1 / 3}
\end{aligned}
$$

Note that the total number concentration does not change during the condensation process, and the two size parameters of the log-normal distribution-geometric mean diameter $\left(d_{g}\right)$ and geometric standard deviation $\left(\sigma_{g}\right)$-are allowed to vary with time (Jung et al., 2002).

Subsequently, from Eqs. (5) and (6), the Ångstrom exponent can be expressed as follows.

$$
\begin{aligned}
\alpha & =-\frac{\log \left(b_{\text {ext } 1} / b_{\text {ext } 2}\right)}{\log \left(\lambda_{1} / \lambda_{2}\right)} \\
& =-\frac{\log \left(\frac{\left.\int_{0}^{d_{p}^{\max }} \frac{\pi d_{p}^{2}}{4} Q_{e x t}\left(d_{p}, \lambda_{1}, m\right) n\left(d_{p}\right) d d_{p}\right)}{\left.\int_{0}^{d_{p}^{\max }} \frac{\pi d_{p}^{2}}{4} Q_{e x t}\left(d_{p}, \lambda_{2}, m\right) n\left(d_{p}\right) d d_{p}\right)}\right.}{\log \left(\lambda_{1} / \lambda_{2}\right)}
\end{aligned}
$$

The Ångstrom exponent for the aerosol dynamic process can be simulated using the Mie theory, by subtituting in Eq. (11) the size distribution parameters from Eq. (8) for coagulation and from Eq. (10) for condensation. The detailed description of the analytic solution during the two processes can be found in literature 
(Jung et al., 2006; Park et al., 2001; Lee et al., 1984; Lee, 1983).

\section{2 The Ångstrom Exponent during Coagulation and Condensation in the Continuum Regime}

During coagulation and condensation, aerosol continuously changes their size as a function of time. Basically, the aerosol extinction coefficient is a function of the size distribution and compositions, which determine the refractive index. During coagulation, the particle number concentration decreases, geometric mean diameter increases, and overall extinction coefficient $\left(b_{\text {ext }}\right)$ decreases (Jung and Kim, 2008). A higher extinction coefficient is observed for wide aerosol distributions with a large geometric standard deviation than for narrow aerosol distributions with a small geometric standard deviation. During condensation, the visi- bility decreases and the overall extinction coefficient $\left(b_{\text {ext }}\right)$ increases (Jung and Kim, 2008).

Fig. 1 shows the change in the size distribution and the Ångstrom exponent during the coagulation process. Both, the analytic and numerical driven solutions are shown for comparison.

The initial values of the size distribution parameters are as follows: geometric standard deviation, 1.2; geometric mean diameter, $0.2 \mu \mathrm{m}$; and total number concentration, $10,000 / \mathrm{cm}^{3} . K N_{0} t$ is the coagulation-based dimensionless time (Lee, 1983).

As Fig. 1 shows, the changes in the Angstrom exponent with the size distribution as derived from analytical and numerical solutions agree very well; note that the "analytic solution" refers to the coagulation and condensation dynamic solution from previous section. Fig. 1 also shows that the Ångstrom exponent decreases during the coagulation process. The large (a) Geometric standard deviation

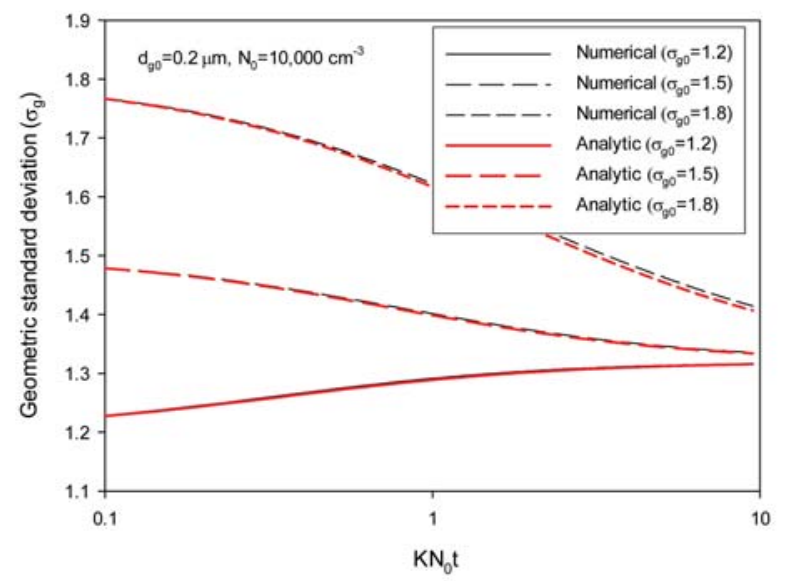

(c) Geometric mean diameter

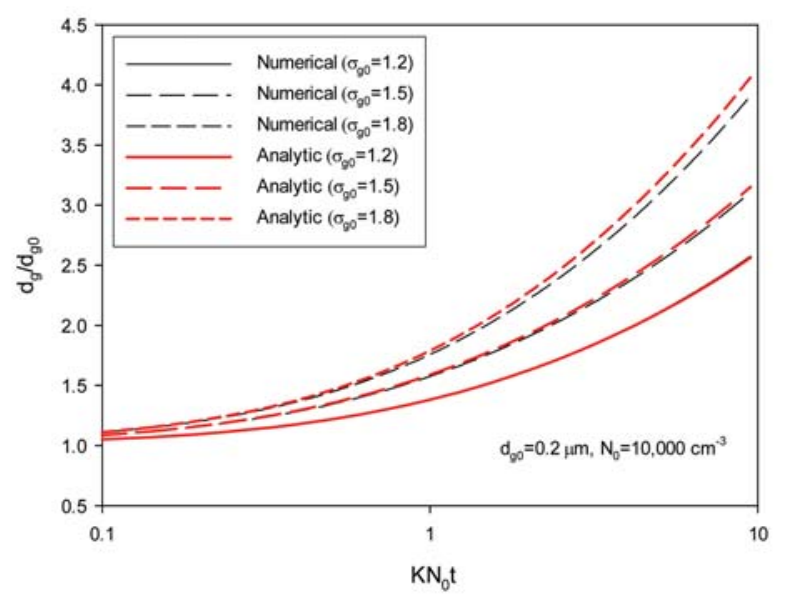

(b) Number concentration

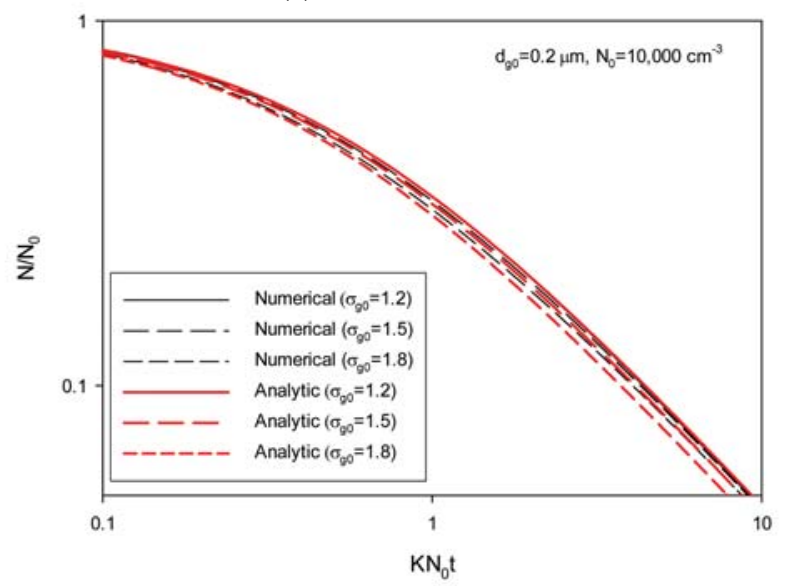

(d) Ångstrom exponent

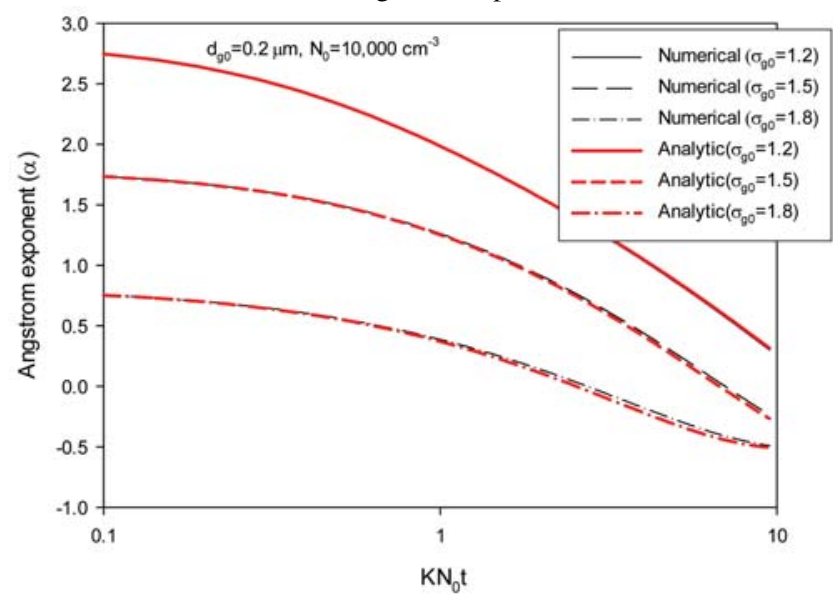

Fig. 1. The changes in the size distribution and the Ångstrom exponent during the coagulation process (Numerical vs. analytic solution, $x$ axis: coagulation-based dimensionless time). 


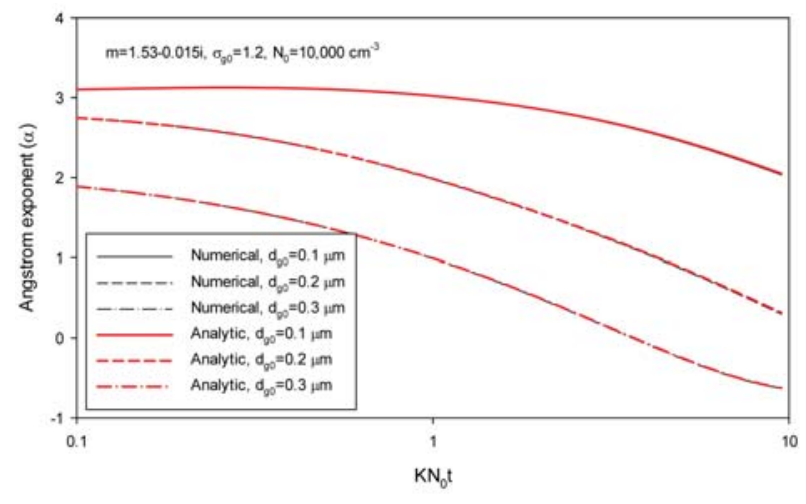

Fig. 2. The changes in the Ångstrom exponent during the coagulation process for different initial geometric mean diameters (Numerical vs. analytic solution, $x$ axis: coagulationbased dimensionless time, $y$ axis: Ångstrom exponent).

(a) Geometric standard deviation

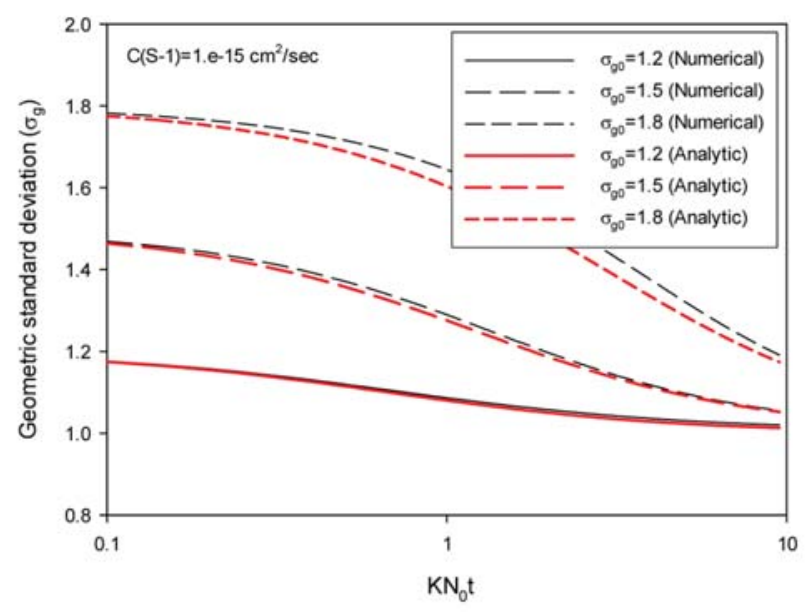

initial geometric standard deviation with wide size distribution corresponds to a larger Ångstrom exponent than does the small initial geometric standard deviation.

Fig. 2 shows the change in the Angstrom exponent with the geometric mean diameter during the coagulation process. The initial values are as follows: geometric standard deviation, 1.2; total number concentration, $10,000 / \mathrm{cm}^{3}$; and refractive index, 1.53-0.015i.

During coagulation, the total number concentration decreases, geometric mean diameter converges to a value around 1.32, and the geometric mean diameter increases (Lee, 1983).

As Fig. 2 shows, the Ångstrom exponent decreases rapidly as the initial geometric mean diameter increases. Fig. 2 also shows that the Ångstrom exponent decreases to a lower value for a large initial geometric mean diameter than for a small initial geometric mean diameter.

(b) Geometric mean diameter

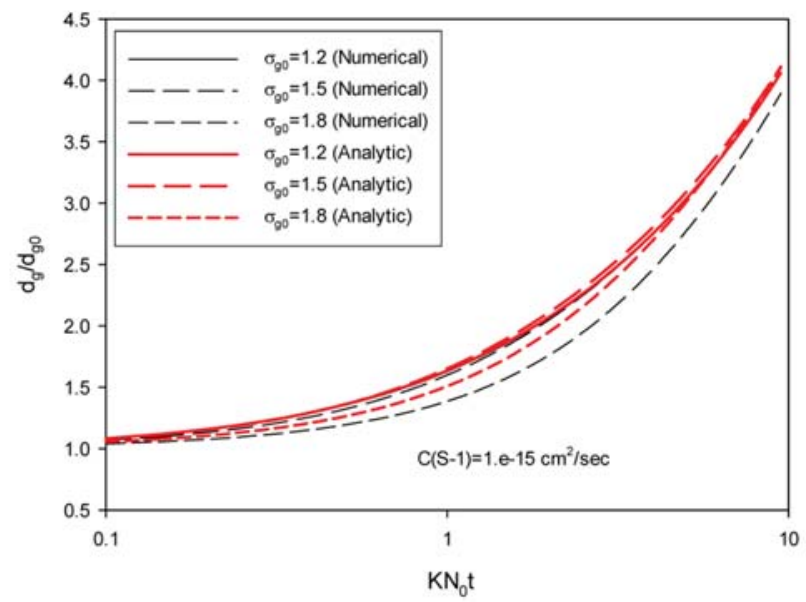

(c) Ångstrom exponent

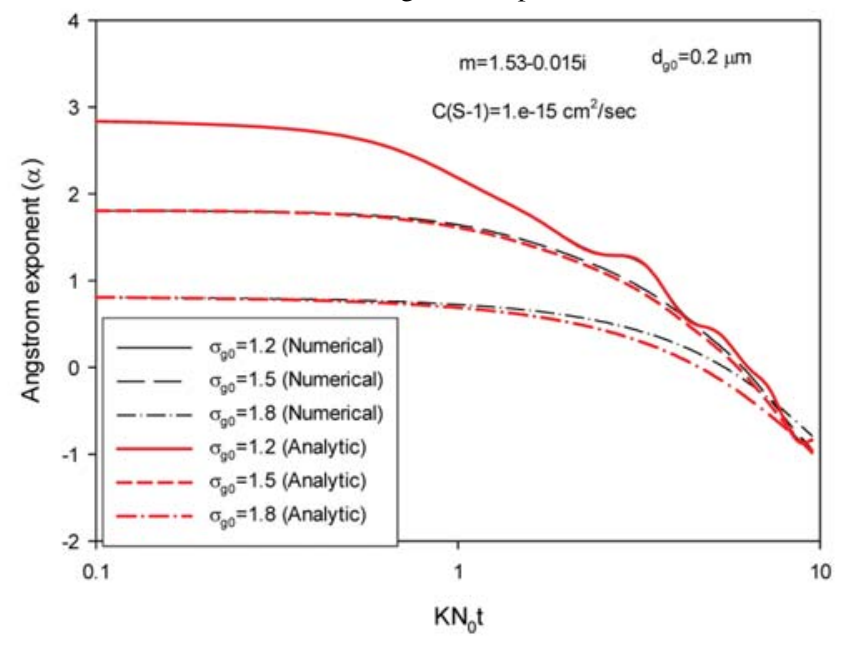

Fig. 3. The changes in the size distribution and the Ångstrom exponent during the condensation process (Numerical vs. analytic solution, $x$ axis: coagulation-based dimensionless time). 
(a) Geometric standard deviation

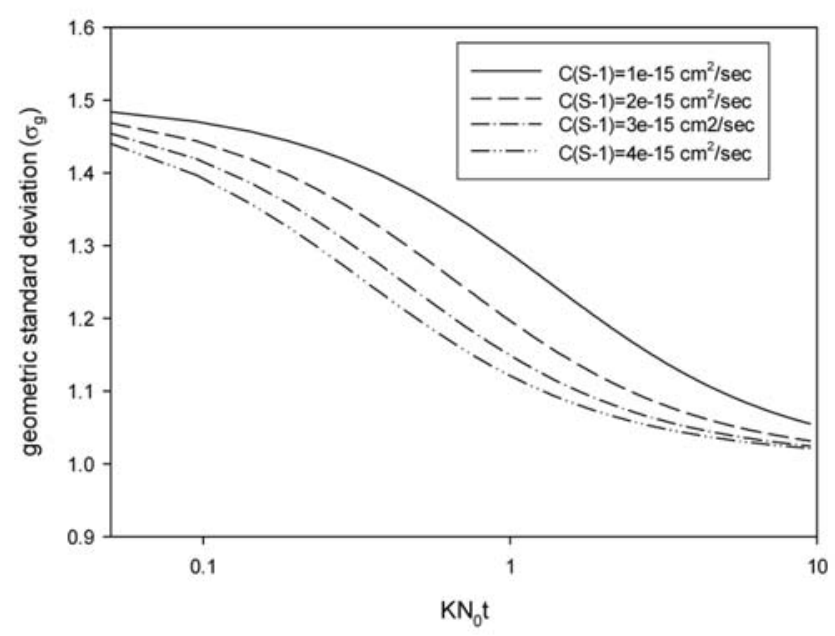

(b) Geometric mean diameter

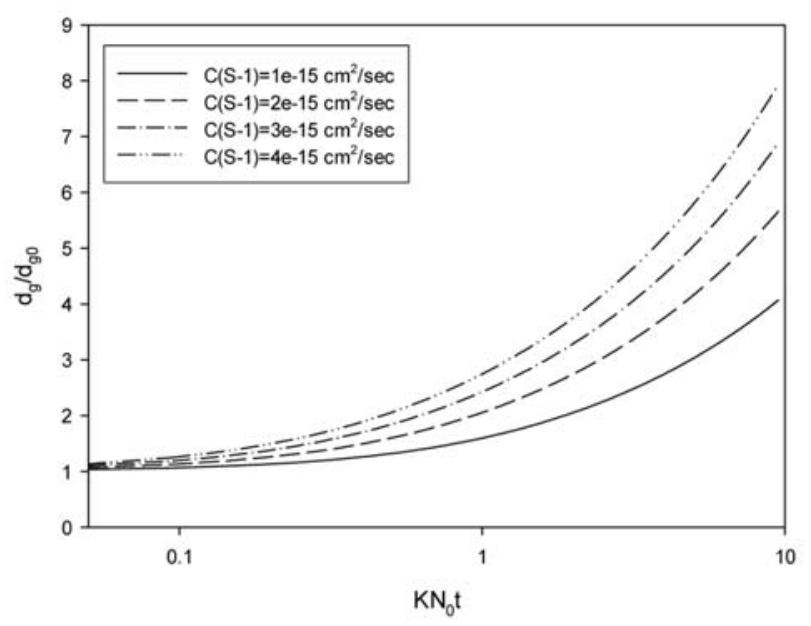

(c) Ångstrom exponent

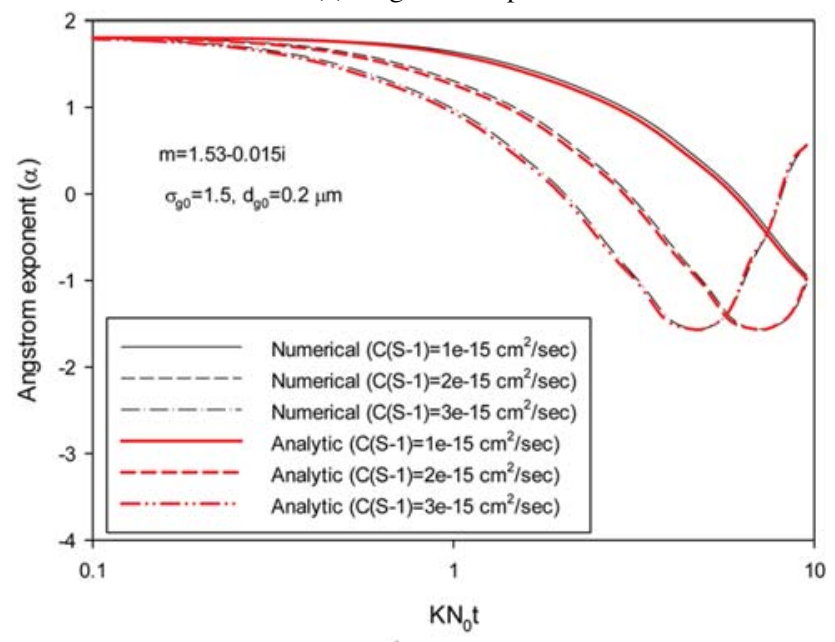

Fig. 4. The changes in the Ångstrom exponent during condensation under different condensation conditions (Numerical vs. analytic solution, $x$ axis: coagulation-based dimensionless time).

Fig. 3 shows the change in the size distribution and the Angstrom exponent during the condensation process. The condensation rate of $1 \mathrm{e}-15 \mathrm{~cm}^{3} / \mathrm{cm}^{3} / \mathrm{s}$ was considered for different initial geometric standard deviation values. We used $K N_{0} t$ for the condensation process as a time variable in order to compare coagulation results for the same time dimension. The Ångstrom exponents derived based on the size parameter from numerical and analytic solutions were compared and showed good agreement. Throughout the condensation process, the number concentration did not change; the geometric mean diameter converged to 1 , and the geometric mean diameter increased. As Fig. 3 shows, the Ångstrom exponent decreases as the initial geometric standard deviation increases. The Ångstrom exponent decreases during condensation, and the rate of decrease in the Ångstrom exponent is larger for small initial geometric standard deviation values. Fig. 3 also shows that the difference in the Angstrom exponent between numerical and analytic solution increase with time. The discrepancy in size parameter estimation between the numerical and analytic results for the condensation process is larger than that for the coagulation process; this is due to the difference between the Angstrom exponents for the numerical and analytic results.

Fig. 4 shows the change in the Angstrom exponent during condensation under different condensation conditions. As Fig. 4 shows, the exponent decreases to a minimum value and then increases as condensation 
(a) $\mathrm{m}=1.53$

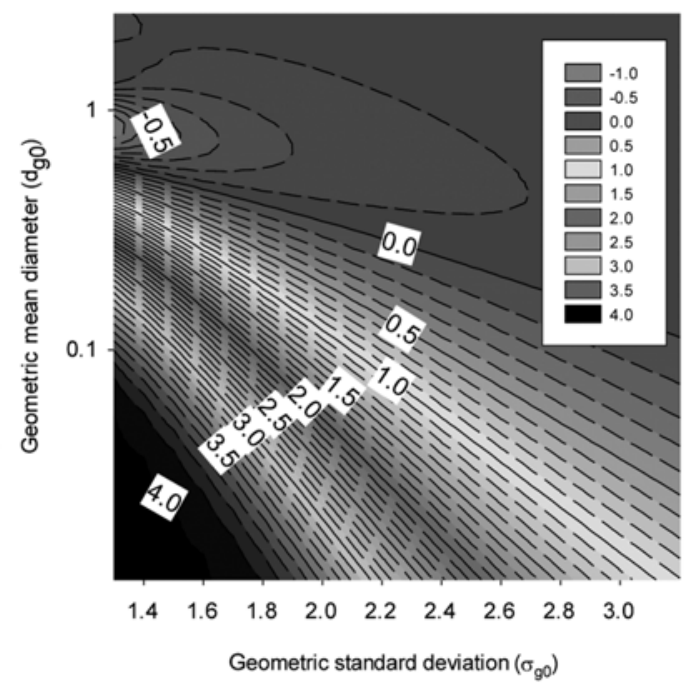

(c) $\mathrm{m}=1.53-0.1 \mathrm{i}$

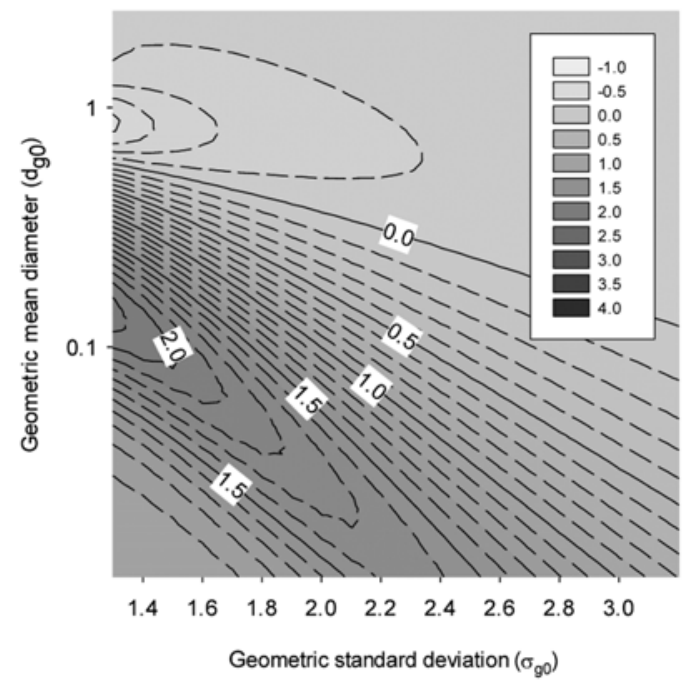

(b) $\mathrm{m}=1.53-0.01 \mathrm{i}$

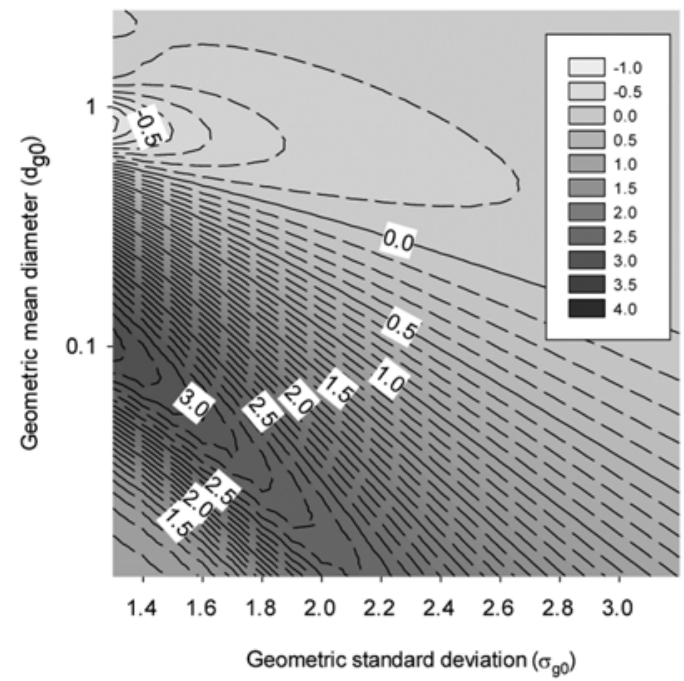

(d) $m=2.0$

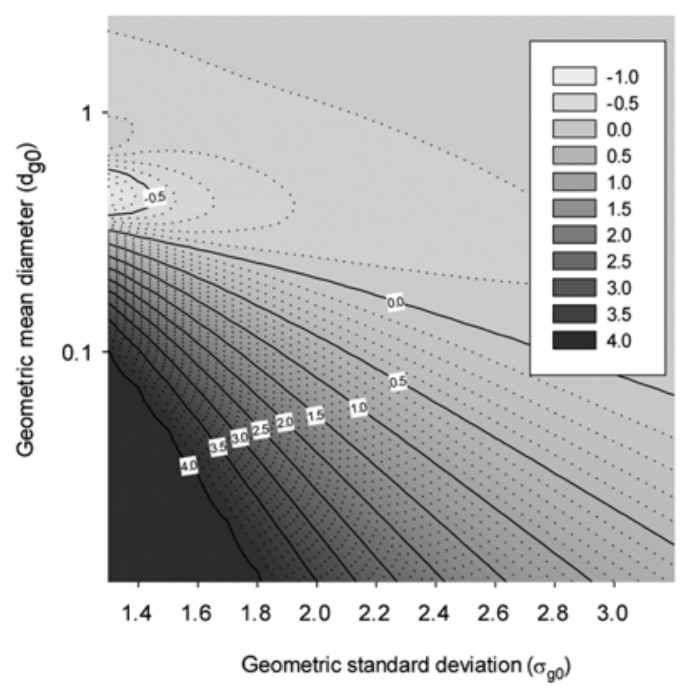

Fig. 5. Sensitivity of the Ångstrom exponent as a function of the geometric standard deviation and geometric mean diameter for different refractive indices ( $x$ axis: geometric standard deviation, $y$ axis: geometric mean diameter).

proceeds. Fig. 4 shows that increasing the condensation rate accelerates these tendencies because the size distribution parameters change more rapidly as the condensation rate $(=\mathrm{C}(\mathrm{S}-1))$ increases.

As Figs. 1-4 show, the change in the Ångstrom exponent is closely related to the refractive index of particles as well as size distribution. Fig. 5 shows the sensitivity of the Ångstrom exponent as a function of geometric standard deviation $\left(\sigma_{g}\right)$ and geometric mean diameter $\left(d_{g}\right)$ for different refractive indices. In Fig. 5, the Ångstrom exponent has been calculated for the same size distribution with different refractive indices. The
Ångstrom exponent approaches 0 for large particles and 1 for small particles with diameters less than 0.01 $\mathrm{mm}$. For nuclei-mode particles with geometric mean diameters less than $0.1 \mathrm{~mm}$, the Angstrom exponent approaches 4 in the case of a real refractive index for nonabsorbing particles. However, for absorbing particles with an imaginary refractive index, the Angstrom exponent approaches 1 (Jung and Kim, 2013); this explains the behavior observed at the right bottom edge of Fig. 5(b). The geometric mean diameter at which the Angstrom exponent has a peak value decreases as the initial geometric standard deviation incr- 

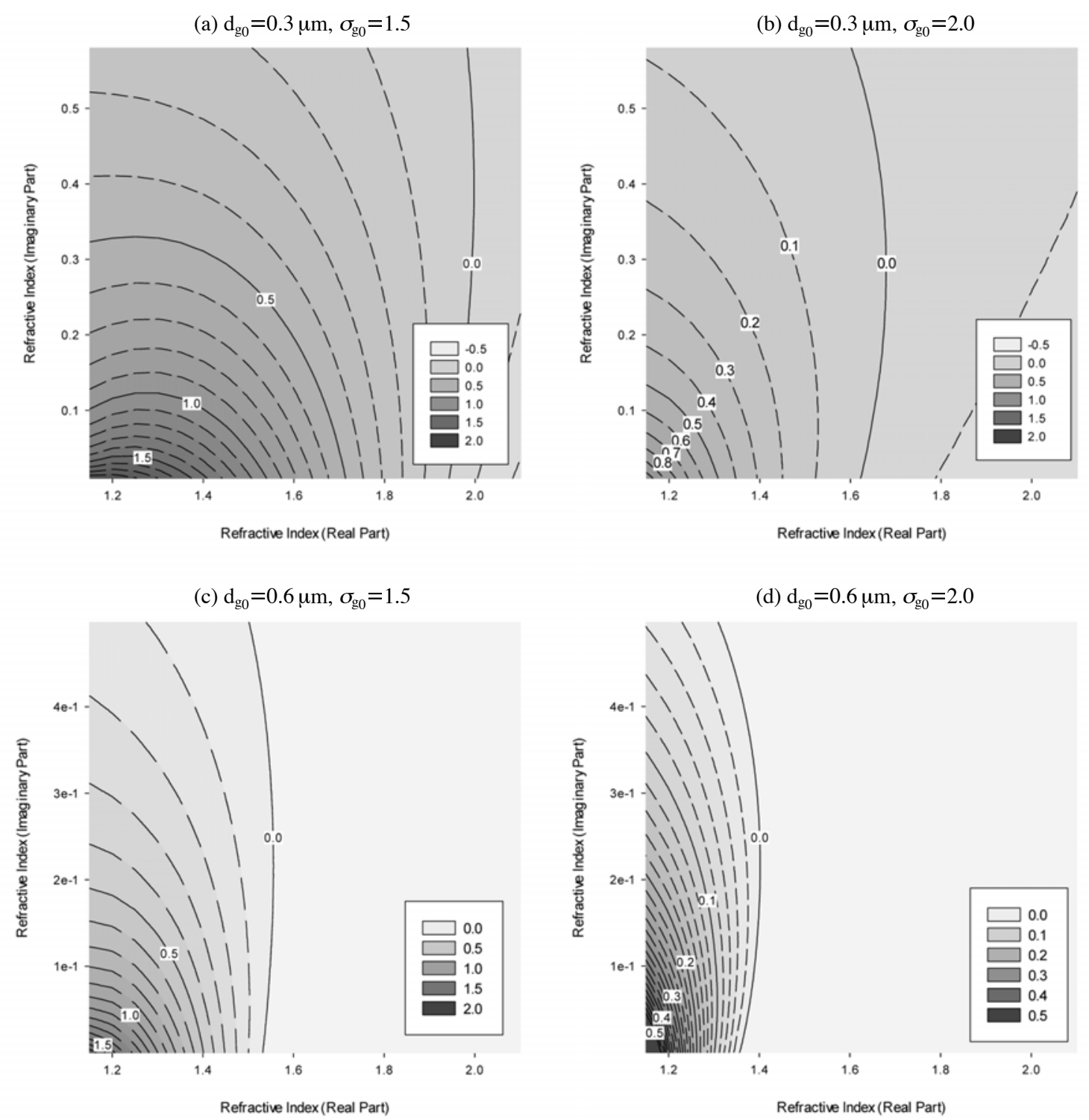

Fig. 6. Sensitivity of the Ångstrom exponent as a function of the real and imaginary parts of the refractive indices for different size parameters ( $x$ axis: the real part of the refractive index, $y$ axis: the imaginary part of the refractive index).

eases and the imaginary part of the refractive index decreases. The peak value decreases as the imaginary part of the refractive index increases (Jung and Kim, 2013).

In Fig. 5(a), (b) and (c), the results for refractive indices $1.53,1.53-0.01 \mathrm{i}$, and 1.53-0.1i are compared to determine the effect of absorbing particles (the imaginary part) on the Ångstrom exponent. In Fig. 5(a) and (d), the results for refractive indices 1.53 and 2 are compared to determine the effects of the real part of the refractive index. As Fig. 5 shows, the Angstrom exponent is sensitive to the refractive index; the figure shows that for nonabsorbing particles, where only real part of refractive index considered, the Ångstrom exponent decreases as the geometric standard deviation and geometric mean diameter increases. For absorbing particles (i.e., taking into account the imaginary part), however, the Ångstrom exponent may increase or decrease depending on the initial geometric standard deviation and geometric mean diameter. Generally, the Ångstrom exponent has a maximum value for a geometric mean diameter of $\sim 0.1 \mu \mathrm{m}$ and small geometric standard deviation. As the geometric standard deviation increases, the geometric mean diameter at which the Ångstrom exponent becomes maximum decreases. 
The size distribution and refractive index are very crucial in estimating the Ångstrom exponent for aerosol dynamic processes.

In order to quantify the effect of refractive index, Fig. 6 also describes the sensitivity of the Ångstrom exponent under different size distributions and refractivity conditions. Fig. 6 shows the sensitivity of the Ångstrom exponent as a function of the real and imaginary part of the refractive indices with different size parameters.

Initial geometric mean diameters of $0.3 \mu \mathrm{m}$ and 0.6 $\mu \mathrm{m}$ with geometric standard deviations of 1.5 and 2.0 were considered. As Fig. 6 shows, the Ångstrom exponent decreases as the geometric standard deviation increases and as the geometric mean diameter increases. Fig. 6 also shows that there exist several pairs of real and imaginary refractive index corresponding to a given Ångstrom exponent. For example, for a geometric mean diameter of $0.3 \mu \mathrm{m}$ and geometric standard deviation of 1.5 , the refractive indices 1.1-0.1i and 1.57 have the same Angstrom exponent of 1.0. This means that the Angstrom exponent and refractive index do not have a one-to-one relation, which makes it difficult to retrieve particle size distribution or particle characteristics from only the measurement of optical properties.

The Ångstrom exponent is a function of aerosol size distribution and aerosol refractive index. The refractive index is related to the chemical composition of the aerosol, and the real and imaginary parts of the refractive index represent the scattering and absorption of aerosol, respectively. During the coagulation and condensation processes, aerosol size distribution changes continuously as a function of time, and this change affects the change in the Ångstrom exponent.

For example, the total number concentration decreases and the geometric mean diameter converges to a value of around 1.32 during coagulation. One the while, the geometric standard deviation approaches 1, indicating that the particle size distribution converges to a monodisperse distribution during the condensation process.

The degree of change in the Angstrom exponent is closely related to the refractive index and initial aerosol size distribution, and it is difficult to pinpoint the parameter to which the Angstrom exponent is more sensitive. However, Figs. 5 and 6 show the degree of change in the Ångstrom exponent as a function of the size parameters such as the geometric mean diameter and geometric standard deviation, and show that the change is more sensitive to the above mentioned size parameters than to refractive index under the simulation conditions.

\section{CONCLUSIONS}

This study shows the Ångstrom exponent for polydispersed aerosol size distribution during the coagulation and condensation processes. Log-normal aerosol size distribution was assumed; moment relations were used; and a sensitive analysis of the Ångstrom exponent during the aerosol dynamic process was conducted.

The change in the Angstrom exponent for different real and imaginary parts of the refractive index was also studied.

The analytic solutions for coagulation and condensation are used, and their results are compared with those corresponding to the numerical solutions. The results show that the Angstrom exponent derived from the two solutions agree well.

The Ångstrom exponent changes sensitively as a function of size distribution and refractive index.

During the coagulation process, the geometric mean diameter increases, the number concentration decreases, and the geometric standard deviation converges to 1.32 (Lee, 1983). During the condensation process, the geometric mean diameter increases, and the geometric standard deviation converges to 1 . These changes in size distribution during the aerosol dynamic process affect the Ångstrom exponent.

The results also show how the Ångstrom exponent changes for different refractive indices during the aerosol dynamics processes, and the results were compared with those obtained based on simple analytic size distribution solutions. Usually, the Ångstrom exponent decreases during aerosol dynamic processes because of the growth in particle diameter. However, the degrees of change depend on the size distribution and refractive index. Subsequently, this study shows that the size parameter and refractive index can be important factors in determining aerosol optical properties. This study considers unimodal size distribution; however, the real atmosphere is known to have multimodal size distribution. It is important to compare the simulation results with measurement data, which can be a subject for future study.

\section{ACKNOWLEDGEMENT}

This work was funded by the Korea Meteorological Administration Research and Development Program under CATER 2012-7070 and by Basic Science Research Program through the National Research Foundation of Korea (NRF) funded by the Ministry of Education, Science and Technology (No. 2012R1A1A2001 133). 


\section{REFERENCES}

Binkowski, F.S., Shankar, U. (1995) The regional particulate matter model. 1: Model description and preliminary results. Journal of Geophysical Research 100, 2619126209.

Cachorro, V.E., De Frutos, A.M. (1994) Retrieval of atmospheric aerosol characteristics from visible extinction data at Valladolid. Atmospheric Environment 28, 963971.

Jung, C.H., Kim, Y.P., Lee, K.W. (2002) Simulation of the influence of coarse mode particles on the properties of fine mode particles. Journal of Aerosol Science 33, 1201-1216.

Jung, C.H., Kim, Y.P. (2006) Numerical estimation of the effects of condensation and coagulation on visibility using the moment method. Journal of Aerosol Science 37, 143-161.

Jung, C.H., Kim, Y.P. (2008) Theoretical study on the change of the particle extinction coefficient during the aerosol dynamic processes. Journal of Aerosol Science 39, 904-916.

Jung, C.H., Kim, Y.P. (2010) Simplified analytic model to estimate Ångstrom exponent in Junge aerosol size distribution. Environmental Engineering Science 27, 789-795.

Jung, C.H., Kim, Y.P. (2013) Analytic solution on the estimation of the Ångstrom exponent in Log-normal aerosol size distribution. Particulate Science and Technology, in press.
Lee, K.W. (1983) Change of particle size distribution during Brownian coagulation. Journal of Colloid and Interface Science 92, 315-325.

Lee, K.W., Chen, H., Gieseke, J.A. (1984) Log-normally preserving size distribution for Brownian coagulation in the free-molecule regime. Aerosol Science and Technology 3, 53-62.

O’Neill, N.T., Eck, T.F., Holben, B.N., Smirnov, A., Dubovik, O. (2001a) Bimodal size distribution influences on the variation of Ångstrom derivatives in spectral and optical depth space. Applied Optics 40, 2368-2375.

O’Neill, N.T., Eck, T.F., Smirnov, A., Holben, B.N., Thulasiraman, S. (2001b) Spectral discrimination of coarse and fine mode optical depth. Journal of Geophysical Research 108, doi:10.1029/2002JD002975.

Park, S.H., Lee, K.W., Shimada, M., Okuyama, K. (2001) Alternative analytical solution to condensational growth of polydisperse aerosols in the continuum regime. Journal of Aerosol Science 32, 187-197.

Schuster, G.L., Dubovik, O., Holben, B.N. (2006) Ångstrom exponent and bimodal aerosol size distributions. Journal of Geophysical Research 111, Do7207, doi: 101029/2005Jd006328.

Seinfeld, J.H., Pandis, S.N. (1998) Atmospheric Chemistry and Physics. Wiley, New York, USA.

(Received 14 September 2012, revised 11 December 2012, accepted 11 December 2012) 